\title{
Attitudes toward advance directives and progno- sis in patients with heart failure: a pilot study
}

\author{
JinShil Kim ${ }^{1}$, Minjeong An ${ }^{2}$, Seongkum $\mathrm{Heo}^{3}$, and Mi-Seung Shin 4
}

\author{
${ }^{1}$ Gachon University College of \\ Nursing, Incheon; ${ }^{2}$ Chonnam \\ National University College of \\ Nursing, Gwangju, Korea; ${ }^{3}$ College \\ of Nursing, University of Arkansas \\ for Medical Sciences, Little Rock, \\ AR, USA; ${ }^{4}$ Department of Internal \\ Medicine, Gil Medical Center, \\ Gachon University College of \\ Medicine, Incheon, Korea
}

Received: April 12, 2018

Revised : July 5, 2018

Accepted: July 5, 2018

\section{Correspondence to} Mi-Seung Shin, M.D.

Division of Cardiology, Department of Internal Medicine, Gil Medical Center, Gachon University College of Medicine, 21 Namdong-daero 774beon-gil, Namdong-gu, Incheon 21565 , Korea

Tel: $+82-32-460-3663$

Fax: +82-32-469-1906

E-mail:msshin@gilhospital.com
Background/Aims: Advance directives (ADs) in Korean patients with heart failure (HF) and the associations of attitude towards ADs and HF prognosis with ADs were initially assessed using the model of the Korean-Advance Directive (K-AD). Methods: Twenty-four patients with HF (age, 67.1 years; men, 58.3\%; ejection fraction, 35.9\%) participated. A pilot test to evaluate the feasibility of ADs and the possible associations of attitudes towards ADs and prognosis with end-of-life treatment preferences among patients with $\mathrm{HF}$ was conducted.

Results: Fifteen patients (62.5\%) completed the K-ADs. The major reason for incomplete K-AD was knowledge deficit. Patients valued "comfortable death" the most (45.4\%), followed by "giving no burden to the family" (13.6\%). Among treatment preferences, hospice care was preferred by the majority $(66.7 \%)$, while cardiopulmonary resuscitation (CPR) was preferred by the minority (31.8\%). Children (50.0\%) were mostly appointed as a proxy, followed by the spouse (33.3\%). More patients with moderately positive attitudes completed the K-ADs than their counterparts (70.0\% vs. $57.1 \%$ ). The 5 -year survival rate was $69.2 \%$; the patients who preferred CPR had a higher survival rate (70.6\% vs. 68.5\%) whereas those who preferred hospice care had a lower survival rate than their counterparts (70.7\% vs. $75.2 \%)$.

Conclusions: The findings support the feasibility of the K-AD model, with a high acceptance rate in two-thirds of the sample. Further studies are warranted to investigate whether treatment preferences are associated with attitude towards ADs and/or HF prognosis using larger sample size.

Keywords: Heart failure; Advance directives; Palliative care; Attitude; Prognosis

\section{INTRODUCTION}

Heart failure (HF), which is one of the most debilitating chronic diseases, is a pandemic disease [1] that affected more than 37.7 million individuals globally in 2010 [2]. Despite therapeutic advances [3], distressing symptoms and economic burden of HF have been escalating worldwide due to the progressive deterioration of the illness $[2,4,5]$. Such burdens mostly arise from frequent emergency visits or hospitalization to treat HF decom- pensation $[4,6]$. Further, despite improvement in the survival rates, the mortality rates remain high $[4,7]$, with the 5-year survival rate after the diagnosis being limited to only about $50 \%$ [4].

One approach to reduce the healthcare burden of HF and improve patient outcomes is the integration of and transition to palliative care $[8,9]$. To ensure continued care through the smooth transition to palliative care, an ongoing discussion on routine care is critical, which focuses on aspects including, but not limited to, periodic 
prognostic discussions, symptom management, complex therapeutic options and their risks/benefits, and other further concerns/issues $[9,10]$. In this process, various forms of advance directives (ADs) could be useful, particularly for patients with end-stage HF under palliative and supportive care [11]. However, earlier introduction is recommended prior to progress to the end-stage condition when clinical improvements via modifying therapies are no longer expected, while initiating endof-life (EOL) considerations [9,12]. When patients actively engage in ongoing discussions regarding their care, they are enabled to prepare their plans for and make shared decisions of EOL care using ADs based on their personal values, treatment preferences, and shared goals of care $[11,12]$.

Emerging evidence largely from western countries has proven the effectiveness of palliative care in HF for clinical outcomes, including symptom improvement [13], fewer rehospitalizations in the EOL period [8,13], and further overall cost savings $[10,14]$. Despite the well-documented need for and clinical benefits of palliative care in $\mathrm{HF}$ [10], patients, even with end-stage disease, have limited access to a timely transition or receive a delayed referral to palliative care $[9,15]$ or suboptimal use of ADs $[16,17]$. The major barriers to underutilized palliative care with ADs for patients with HF were unpredictable prognosis, knowledge deficit or poor awareness, communication difficulty with a lack of model that is fit for non-hospice palliative care, or reluctance to have such a discussion $[15,18,19]$. While empirical evidence about modifiable factors which could facilitate written ADs or decisions on EOL medical care still remain insufficient, an available patient data-based study showed that favorable attitudes following a palliative consultation for patients with advanced HF possibly increased the completion of ADs [20]. In Korea, despite the increased awareness for and attention to the importance of integrated palliative care in HF, little research has been conducted. The only evidence found was that Korean healthcare professionals in cardiovascular care agreed on palliative care for patients with HF, particularly those in the advanced stage [11].

Therefore, as an initiative, this preliminary study was conducted to explore the feasibility of ADs for patients with HF in Korea. Its specific aims included: (1) exploration of the reasons for completing or not completing
ADs, using the Korean-Advance Directive (K-AD) model, (2) exploration of the EOL values, treatment preferences, and proxy appointments of patients with HF using the $\mathrm{K}-\mathrm{AD}$ model, (3) examination of the association between attitudes toward ADs and $\mathrm{K}-\mathrm{AD}$ model completion in patients with $\mathrm{HF}$, and (4) examination of the association of each of the four EOL treatment preferences (cardiopulmonary resuscitation [CPR], artificial ventilation, hemodialysis, and hospice use) with the prognosis of patients with $\mathrm{HF}$.

\section{METHODS}

\section{Design and procedure}

A pilot test to evaluate the feasibility of ADs and the possible associations of attitudes towards ADs and prognosis with EOL treatment preferences among patients with HF was conducted. Patients with HF who visited the outpatient clinic for routine care were enrolled. This study was approved by the Institutional Review Board of Gachon University Gil Medical Center (GBIRB2017-058), and all patients provided informed consent prior to face-to-face interviews for data collection.

As part of the usual care, a physician assessed the patient prognosis based on their individual conditions and treatment responses. This was followed by an introduction to the study regarding palliative care survey, including attitude towards $\mathrm{AD}$ and $\mathrm{K}-\mathrm{AD}$ model usage. Thereafter, a trained research coordinator conducted face-to-face interviews for these surveys. If the patients agreed, a study coordinator assisted them to prepare the $\mathrm{K}-\mathrm{AD}$ model; if this option was rejected, the reasons for their decision were asked.

\section{Participants}

Patients with HF were enrolled if they met the following criteria: (1) age of $\geq 18$ years; (2) disease duration of $\geq 6$ months after HF diagnosis; (3) guideline-directed medical therapy, including beta-blockers, angiotensin-converting enzyme inhibitors (ACEIs), angiotensin receptor blockers (ARBs), and diuretics; and (4) willingness to participate in a discussion regarding their own illness trajectory. Patients were excluded if they met the following criteria: (1) had documented neuropsychological disorders, involving substantial cognitive impairments, 
which could possibly limit the understanding of the study protocol, such as dementia or Alzheimer's disease, (traumatic) brain disorders, stroke, or psychiatric disorders; (2) a candidate of heart transplantation; (3) having terminal comorbid condition(s) requiring life-sustaining treatments (LSTs), such as terminal cancer, acquired immune deficiency syndrome, chronic obstructive pulmonary disease, or liver cirrhosis [21]; (4) under care from a hospice care team; or (5) presence of a left ventricular assist device.

\section{Measures}

In this preliminary study, the patients with HF completed the following measures: $\mathrm{AD}$, attitudes toward $\mathrm{ADs}$, and estimation of HF prognosis.

\section{Advance directive}

The K-AD model was a measure of an $\mathrm{AD}$, which was developed to explore EOL values, treatment directives, and preferred surrogate decision-makers [22,23]. For the personal value statement, the patients were asked to state their EOL values freely. For the treatment directives, the patients' decisions regarding EOL treatment preferences were asked, assuming one's incapability for such a decision-making. This section originally included four items (CPR, artificial ventilation, tube feeding, and hospice care). It was revised after the Act on Hospice and Palliative Care and Decisions on Life-Sustaining Treatment for Patients at the End of Life [21], with a malignancy version and a non-malignancy version; an additional item of "chemotherapy" was included for the malignancy version and the replacement of tube feeding with "hemodialysis" was included for both versions. The patients were also asked to appoint a proxy if available. They were also informed that such a document could be completed in part with modifications periodically through ongoing discussions.

Completion of the K-AD model was optional. The patients were asked if they were interested in writing the $\mathrm{K}-\mathrm{AD}$ model on a dichotomous scale $(1=$ yes, $\mathrm{O}=\mathrm{no})$. They were then asked to state the reasons for their refusal or agreement to complete the K-AD model.

\section{Attitude toward advance directives}

The attitudes were assessed using the 16-item Advance Directive Attitude Survey (ADAS) [24]. The extent to which the patient's views regarding illness perceptions, opportunities for treatment choices, effects of ADs on treatment, and impact of ADs on the family were positive or negative was determined on a four-point Likert scale ( $1=$ strongly disagree to $4=$ strongly agree). The possible scores range from 16 to 64 , with higher scores indicating more positive attitudes towards AD. Psychometric properties were reported previously with a reliability coefficient within the desirable limit of alpha of 0.74 [24]. A Korean translated version of the scale (K-ADAS) also showed a desirable reliability with a Cronbach's alpha of 0.79 among elderly people from senior centers [25].

\section{HF prognosis}

The Seattle Heart Failure Model (SHFM) is a prognostic risk score model for the estimation of HF prognosis [26,27]. The SHFM provides 1-, 2-, and 5-year survival (mortality) estimates based on the clinical status, medications, laboratory data, and device therapy [26]. The accuracy of the model as an assessment tool for HF prognosis was well documented [27,28].

\section{Statistical analysis}

IBM SPSS for Windows version 23.0 (IBM Co., Armonk, NY, USA) was used for statistical analysis. The level of statistical significance was set at a $p$ value of 0.05 . Descriptive statistics were conducted to describe the sample; the results were presented as frequencies, percentages, and means and standard deviations. Depending on the level of the measurements of the study aims, non-parametric tests were conducted for these preliminary tests, including Mann-Whitney U test and Kruskal-Wallis test for group comparisons and chi-square analysis for the assessment of associations.

\section{RESULTS}

Twenty-four patients with HF were enrolled and participated in this study (mean age, 67.1 \pm 9.9 years; male sex, 14 [58.3\%]). The duration of HF diagnosis was $103.8 \pm 52.9$ months (range, 13.0 to 208.0); the comorbid index score was $1.6 \pm 0.6$ points. The etiologies of HF were dilated cardiomyopathy ( $\mathrm{n}=10,41.7 \%)$, ischemic cardiomyopathy $(\mathrm{n}=9,37.5 \%)$, atrial fibrillation $(\mathrm{n}=3,12.5 \%)$, and valvular heart disease $(\mathrm{n}=2,8.3 \%)$. The mean left ven- 
tricular ejection fraction (LVEF) was 35.9\%. One patient was asymptomatic (New York Heart Association [NYHA] Class I); most patients had mild (NYHA Class II, $\mathrm{n}=$ 19, 79.2\%) and moderate (NYHA Class III, $\mathrm{n}=4,16.7 \%$ ) HF. The patients with HF were on optimal medication, ACEIs (50.0\%), ARBs (45.8\%), beta-blockers (75.0\%), and diuretics (33.3\%). Regarding the ADs, six patients (25.0\%) responded that they were aware of what $\mathrm{ADs}$ are; only two patients had prior experience of ADs for a parent's and a friend's health conditions, respectively (Table 1).

\section{Completion of the K-AD model}

Fifteen patients $(62.5 \%)$ completed the three-component K-AD model; nine (37.5\%) were not able to complete. Among the nine patients who did not complete the K-AD model, six patients provided reasons, including "do not know about ADs" ( $\mathrm{n}=5)$ and "difficulty in understanding of the ADs and/or deciding EOL care or understanding" $(n=1)$. The distributions of age, sex, and educational status were similar between those who completed the K-AD model and those who did not. Clinical characteristics regarding the LVEF (Mann-Whitney $U$ test, $p=0.450$ ) and NYHA classes (NYHA classes I/II and III, $\chi^{2}=0.3, p=0.572$ ) were also not significantly different between the two groups; despite the insignificant difference, the patients who completed the K-AD model had a lower LVEF (34.3\% vs. 38.4\%) and greater HF severity (NYHA III, 75.0\% vs. 60.0\%) than those who did not.

\section{K-AD value statement, treatment preferences, and proxy appointment}

Patients with HF who completed the K-AD model valued "comfortable death (with family members) during EOL moment" the most ( $\mathrm{n}=10,45.4 \%)$, followed by "giving no burdens to their family including children" ( $\mathrm{n}=$ 3, 13.6\%) (Table 2). Among the four treatment directives, hospice care ( $n=14,66.7 \%)$ was preferred by the majority of the patients who completed the K-AD model, while futile LSTs were preferred less, including CPR (n $=7,31.8 \%$ ), artificial ventilation, and hemodialysis (each $\mathrm{n}=2,9.5 \%)$. A child, either a son or a daughter $(\mathrm{n}=12$, $50.0 \%$ ), was mostly appointed as a proxy, followed by a spouse (a wife or a husband) ( $\mathrm{n}=8,33.3 \%$ ).

Further, patients with HF who were younger (age $<65$ years: $33.3 \%$ vs. $30.8 \%$ ), female (40.0\% vs. $25.0 \%$ ), and had greater HF severity (NYHA Class III, $50.0 \%$ vs. NYHA
Table 1. Demographic and clinical characteristics of the patients with heart failure $(n=24)$

\begin{tabular}{|c|c|c|}
\hline Variable & Value & Range \\
\hline Age, yr & $67.08 \pm 9.94$ & $50.00-88.00$ \\
\hline Male sex & $14(58.3)$ & \\
\hline Marital status (married) & $17(70.8)$ & \\
\hline Education, yr & $8.78 \pm 4.37$ & $0-16$ \\
\hline$<12$ & $16(66.6)$ & \\
\hline 12 & $3(12.5)$ & \\
\hline$>12$ & $4(16.7)$ & \\
\hline $\begin{array}{l}\text { Heart failure duration, } \\
\text { mo }\end{array}$ & $103.79 \pm 52.85$ & $13-208$ \\
\hline $\begin{array}{l}\text { Left ventricular } \\
\text { ejection fraction, \% }\end{array}$ & $35.88 \pm 8.95$ & $13.00-49.00$ \\
\hline \multicolumn{3}{|l|}{ NYHA classes } \\
\hline I & $1(4 \cdot 2)$ & \\
\hline II & $19(79.2)$ & \\
\hline III & $4(16.7)$ & \\
\hline IV & 0 & \\
\hline \multicolumn{3}{|l|}{ Etiology } \\
\hline DCM & $10(41.7)$ & \\
\hline ICM & $9(37 \cdot 5)$ & \\
\hline AFib & $3(12.5)$ & \\
\hline VHD & $2(8.3)$ & \\
\hline $\begin{array}{l}\text { Charlson comorbidity } \\
\text { index }\end{array}$ & $1.59 \pm 0.59$ & $1-3$ \\
\hline \multicolumn{3}{|l|}{ Medication (yes) } \\
\hline ACE inhibitor & $12(50.0)$ & \\
\hline $\mathrm{ARB}$ & $11(45 \cdot 8)$ & \\
\hline Beta-blockers & $18(75.0)$ & \\
\hline Loop diuretics ${ }^{\mathrm{a}}$ & $8(33 \cdot 3)$ & \\
\hline AD awareness (yes) & $6(25 \cdot 0)$ & \\
\hline $\begin{array}{l}\text { Previous experience with } \\
\text { AD (yes) }\end{array}$ & $2(8.3)^{b}$ & \\
\hline Need for ADs (yes) & $4(20.0)$ & \\
\hline
\end{tabular}

Values are presented as mean \pm SD or number (\%).

NYHA, New York Heart Association; DCM, dilated cardiomyopathy; ICM, ischemic cardiomyopathy; AFib, atrial fibrillation; VHD, valvular heart disease; ACE, angiotensin-converting enzyme; ARB, angiotensin receptor blocker; $\mathrm{AD}$, advance directives.

${ }^{a}$ Includes furosemide or torsemide.

${ }^{\mathrm{b}}$ Reported a previous AD experience with either a parent or others. 
Table 2. Korean-Advance Directive value statements of the patients

\begin{tabular}{lc}
\hline Value statements & No. of patients $(\%)$ \\
\hline Die comfortably during end-of-life & $7(31.8)$ \\
Die comfortably with family members during end-of-life & $3(13.6)$ \\
Give no burdens to the family including children & $3(13.6)$ \\
Leave a living will after looking at all the children & $1(4.5)$ \\
Wait for end-of-life at home & $1(4.5)$ \\
Nothing & $1(4.5)$ \\
Never thought about it & $3(13.6)$ \\
Do not know & $3(13.6)$ \\
Total responses & 22 \\
\hline
\end{tabular}

Table 3. Sample characteristics based on the treatment wishes on the Korean-Advance Directive model

\begin{tabular}{lcccc} 
Characteristic & No selection & $\begin{array}{c}\text { Preferred selections for either } \\
\text { EOL treatments or hospice }\end{array}$ & $\begin{array}{c}\text { Both selections for EOL } \\
\text { treatments and hospice }\end{array}$ & $p$ value \\
\hline Age, yr & $65.7 \pm 6.9$ & $66.5 \pm 8.9$ & $61.2 \pm 10.4$ & 0.440 \\
Education, yr & $9.2 \pm 4.1$ & $10.2 \pm 3.4$ & $6.0 \pm 6.8$ & 0.595 \\
Male sex & $5(83.3)$ & $6(60.0)$ & $3(60.0)$ & 0.333 \\
Marital status (married) & $4(66.7)$ & $9(90.0)$ & $4(80.0)$ & 0.355 \\
Living with someone $^{\mathrm{b}}$ & $3(50.0)$ & $8(80.0)$ & $3(60.0)$ & 0.389 \\
NYHA (I/II) & $5(83.3)$ & $9(90.0)$ & 0.372 \\
\hline
\end{tabular}

Values are presented as mean \pm SD or number (\%). Kruskal-Wallis tests were used for the continuous variables and chi-square tests for the categorical variables.

EOL, end-of-life; NYHA, New York Heart Association functional class.

${ }^{\text {a }}$ Marital status: married vs. other.

${ }^{\mathrm{b}}$ Living with someone: live with someone vs. other.

Classes I/II, 27.8\%) were more likely to choose futile LSTs (i.e., CPR) than their counterparts, although the difference was statistically insignificant. Conversely, the patients in both age groups (66.7\% vs. $66.7 \%)$, who were female $(87.5 \%$ vs. $53.8 \%$ ), and had less severe HF (NYHA classes I/II, 75.0\% vs. NYHA class III, 64.7\%) preferred hospice care. No significant patterns emerged in the demographic and clinical characteristics of the patients who had a preferred choice for an aggressive treatment or hospice care, both choices, or no choice. However, the patients who had a preferred choice for both an aggressive treatment and hospice care were younger, had a lower educational status, were predominantly women and unmarried, and had more severe HF than the patients with either EOL treatments or hospice care, or no choices (Table 3).

\section{Associations of the AD attitude and HF prognosis with the completion of the K-AD model}

Of the 24 patients, 10 patients $(41.7 \%)$ completed the ADAS, with a mean score of $49.20 \pm 4.37$ points (range, 42.00 to 54.00 ). Six patients completed less than $25 \%$ of the items owing to the difficulty in responding. Eight patients did not provide answers to one to three items. The most frequently missing items were those concerning the $\mathrm{AD}$ impact on the family subscale: "My family wants me to have an $\mathrm{AD}$ " ( $\mathrm{n}=7)$; "I think my family would want me to have an advance directive" ( $\mathrm{n}=5$ ); "Making my endof-life treatment wishes clear with an $\mathrm{AD}$ would help to prevent guilt in my family" ( $\mathrm{n}=4$ ); and "Having an AD would make sure that my family knows my treatment wishes" ( $n=4)$. The patients who completed the ADAS demonstrated moderately positive attitudes toward ADs, with their completion of the K-AD model being higher 
Table 4. End-of-life treatment wishes in the Korean-Advance Directive model and predicted survival

\begin{tabular}{|c|c|c|c|}
\hline Treatment wishes & Patients & 5-yr survival, \% & $p$ value \\
\hline CPR & 22 & & 0.689 \\
\hline No & $15(68.2)$ & $68.5 \pm 15.1$ & \\
\hline Yes & $7(31.8)$ & $70.6 \pm 16.0$ & \\
\hline Artificial ventilation & 21 & & 0.947 \\
\hline No & $19(90.5)$ & $70.0 \pm 13.5$ & \\
\hline Yes & $2(9.5)$ & $66.5 \pm 21.9$ & \\
\hline Hemodialysis & 21 & & 1.000 \\
\hline No & $19(90.5)$ & $67.6 \pm 14.1$ & \\
\hline Yes & $2(9 \cdot 5)$ & $66.5 \pm 21.9$ & \\
\hline Hospice care & 21 & & 0.659 \\
\hline No & $7(33 \cdot 3)$ & $75.2 \pm 7.3$ & \\
\hline Yes & $14(66.7)$ & $70.7 \pm 13.3$ & \\
\hline
\end{tabular}

Values are presented as number (\%) or mean \pm SD. Mann-Whitney $U$ tests were used for the comparisons of mean differences of heart failure prognosis in the two groups.

CPR, cardiopulmonary resuscitation.

than that of those who did not (70.0\% vs. 57.1\%).

\section{Relationships of the HF prognosis with the treat- ment preferences}

The 5-year mortality estimation (\%) of this sample size was $30.8 \%$, which was not significantly associated with the K-AD model completion. However, the patients who completed the K-AD model showed a better prognostic estimation ( $\mathrm{n}=15$; mortality, 29.3\%) than those who did not ( $\mathrm{n}=7$; mortality, 34.0\%) (Mann-Whitney $U$ test, $p=$ o.630). The patients who preferred to receive CPR had a higher prognosis, as estimated by the SHFM 5-year survival rate, than their counterparts (70.6\% vs. $68.5 \%$ ). Although the difference was insignificant, the patients who preferred hospice care had a lower 5-year survival rate than their counterparts (70.7\% vs. 75.2\%) (Table 4).

\section{DISCUSSION}

This study initially investigated palliative care of patients with HF in Korea. ADs (K-AD model) were preliminarily explored in the patients with $\mathrm{HF}$, with approximately one in three patients completing the K-AD model. This finding supported the feasibility of the usage of the $\mathrm{K}-\mathrm{AD}$ model in patients with HF. Moderately favorable attitudes towards $\mathrm{AD}$ were noted, with more positive at- titudes associated with more frequent completion of the $\mathrm{K}-\mathrm{AD}$ model. A better prognosis was also associated with more frequent completion of the K-AD model and more choices of futile LSTs, such as CPR, while fewer choices for hospice care in this pilot test.

$\mathrm{ADs}$ in palliative care have been largely employed for patients with cancer, while ADs for non-malignant chronic diseases, including $\mathrm{HF}$, have received less attention [19,29]. In Western countries, integration of or timely transition to palliative care for patients with $\mathrm{HF}$, particularly at the advanced stage, has received increasing attention over the past decade [10,29]. However, access or timely referral to palliative care and/or $\mathrm{AD}$ documentation in $\mathrm{HF}$ are underutilized [10,17], with approximately $4 \%$ of a minor group of patients having access to such care [30]. In Korea, cardiovascular experts initially supported and reached a consensus on palliative care among patients with advanced HF [11]. Any form of ADs is likely to facilitate transitional palliative care in $\mathrm{HF}$ in ongoing therapeutic and palliative discussions on the routine care of $\mathrm{HF}[11,16,17]$.

This aspect of HF care received primary attention in this study, with approximately two-thirds of the patients with HF having favorable attitudes toward ADs and voluntarily providing $\mathrm{AD}$ information using the $\mathrm{K}-\mathrm{AD}$ model, while $37.5 \%$ declining to complete the K-AD model owing to a lack of knowledge and poor understanding 
of $\mathrm{ADs}$ and/or difficulty in making EOL decisions for care. In another study in Korea, $61.1 \%$ of older Korean adults who were recruited from senior centers (mean age, 74.2 years) agreed to prepare ADs if available [25]. Further, 33.1\% of older patients with cancer (mean age, 70.8 years) were aware of ADs in Korea and most patients were willing to complete ADs (93.1\%), if available [31]. In the United States, $67.6 \%$ of elderly Americans, who died between 2000 and 2006, actually had ADs either as living wills or a durable power of attorney [32], while $12.7 \%$ of hospitalized patients with $\mathrm{HF}$ had documented ADs [16]. The patients with HF with demographic patterns associated with more documented ADs were found to be older (> 65 years), female, unmarried, Caucasian, and have a higher socioeconomic status [16]. Despite the initial introduction of ADs among patients with $\mathrm{HF}$ in Korea, following the enactment of the LST Act for individuals with and without terminal conditions [21], these preliminary findings support the feasibility of earlier incorporation of ADs in non-hospice palliative care for patients with HF. Further, a larger study is warranted to assess the demographic and clinical characteristics of patients to increase the awareness and documentation of ADs in Korea.

The specific responses to each component of the K-AD model in the patients with HF were similar to those of other Korean populations with and without cancer [23,33], particularly those of community-dwelling elderly individuals [23]. Comfortable death (31.8\%) and reluctance to burden the family with their care (13.6\%) were highly valued among the patients with HF. Among the community-dwelling elderly individuals in Korea, 35\% valued comfortable death the most, and $28.6 \%$ valued no burden to the family [23]. Also, comfortable death (57.9\%) and no burden to the family (24.3\%) were valued by healthy adults. Most patients with cancer (73.8\%) and their caregivers $(66.7 \%)$ valued comfortable death more, primarily with no pain and/or no burden to the family [33]. Regarding the EOL futile LSTs, approximately one in three patients with $\mathrm{HF}$ (31.8\%) showed preference for CPR, and only two patients showed preference for artificial ventilation or hemodialysis. Although the difference was insignificant, the patients who were younger, female, and had more severe HF were willing to receive CPR, while those who were female and had less severe HF preferred hospice care. Further, the patients of young- er age, lower educational status, female sex, without spouse, and having more severe HF preferred to receive both an aggressive treatment and hospice care in contrast to those with no choices or either EOL treatments or hospice care in this study. In other studies in Korea, CPR was less preferred by patients with cancer-caregiver dyads (20.5\% vs. $27.3 \%)$ and artificial ventilation $(20.5 \%$ vs. $36.4 \%$ ) [33]. In community-dwelling elderly individuals, CPR (23.3\%) and artificial ventilation (24.0\%) were similarly selected [23]. Further, elderly Americans who had documented $\mathrm{AD}$ preferences were more likely to receive limited care, as indicated by their preference, than those without ADs [32].

Such ADs in palliative care of patients with HF are likely under the influence of cultural and/or ethnic circumstances [34]. Our findings initially provide insights into the $\mathrm{AD}$ needs of patients with a non-malignancy condition, i.e., HF in this study; this would possibly facilitate a better understanding of palliative care for Korean patients with HF. In patient-centered care particularly under Confucian beliefs, family members influence an individual's personhood in terms of practices of patient autonomy upon the provision of one's palliative care and/or EOL medical decisions, while preserving individuality [35]. Particularly, appointed proxies regarding EOL medical decisions are largely established by their descendants, particularly the eldest son in Confucian-rooted traditions, highly influencing the parent EOL moment [36]. In this study, half of the patients with HF appointed their adult children, on whom patients would willingly depend, as their surrogate decision-maker to determine or follow their EOL wishes/choices, followed by their spouse. Most community-dwelling elderly individuals also designated their descendants as their proxy (77.1\%), followed by their spouses (17.5\%) [23]. In cancer, $55.0 \%$ of patients and $41.0 \%$ of their spouses designated themselves as a proxy [34]. These findings suggest that the specific features of EOL care on the K-AD model were similar to those of the community-dwelling elderly individuals but were slightly different from those in other malignancies. Further, earlier introduction to ADs is then feasible among patients with $\mathrm{HF}$, with consideration for cultural traditions in the provision of palliative care among them.

Despite the recommendation and establishment of 
palliative practice among patients with HF in western countries, several barriers still challenge such care with the underuse of AD documentation. These barriers to suboptimal palliative care and/or use of ADs involve time conflict, professional training, and resources largely associated with unpredictable HF prognosis, communication difficulty, or a lack of model/system for palliative care $[9,12,29]$. Moreover, negative attitudes toward a care paradigm are also among the major barriers, while favorable attitudes are likely to support palliative care in the context of HF. In one study following a palliative consultation for patients with symptomatic HF (NYHA Classes II/III), attitudes toward ADs improved. Despite the insignificant results, the completion of ADs increased [20]. Such attitudes could be possible in ethnic differences. One study reported that Malays were less willing to participate in advance care planning discussion than Chinese and Indians [37]. In this study, the relationships of the $\mathrm{AD}$ attitudes and prognosis of $\mathrm{HF}$ with the completion of the K-AD model and each of the four treatment directives, respectively, were preliminarily examined. The attitudes toward ADs of the Korean patients with HF were moderately positive (mean, 49.20), which was comparable to the scores in the ADAS of the community-dwelling elderly Koreans from senior centers (mean, 45.12) [25] and older patients with cancer (mean, 48.29) [31]. The patients with positive attitudes also provided more responses to the K-AD model than those who did not complete the ADAS, implying that enhancing $\mathrm{AD}$ attitudes could possibly facilitate the use of ADs in Korea.

Furthermore, HF prognosis was explored for its probable relationships with the EOL treatment preferences. Owing to the nature of a pilot test, the patients with and without choices for CPR had no differences; only two patients had choices for other aggressive treatments, limiting the statistical analyses. However, the patients with a poor prognosis had fewer wishes for CPR and more wishes for hospice care than their counterparts. Previously, fewer than half of the patients with advanced HF completed their ADs; those who had ADs were less likely to receive aggressive care, such as ventilation support or intensive unit care, during their EOL stage [17]. Palliative consultation also likely increases comfort care selection. In one study, patients with advanced HF who were hospitalized for acute decompensation (mean age,
68 years; 91.8\% of African Americans) selected more comfort care (hospice and/or "do not resuscitate" order) than the usual care group 3-6 months after palliative consultation, despite the result being insignificant [38]. Further empirical evidence on palliative care benefits is needed based on the understanding of the circumstance under which the patient makes a decision to give up on prolonged life support.

Owing to the nature of the study, involving a preliminary description using a small sample size, a major limitation arises, suspending any conclusion until results are verified using a larger sample size. In addition, a convenient sample can cause selection biases, which can adversely impact the study validity.

This study raises increased attention to and provides important implications for non-malignancy palliative care for Korean patients with HF in research and practice. More empirical evidence is warranted to explore palliative care awareness, needs, preferences, and benefits/barriers for palliative care among all patients/families and healthcare professionals and their experience during the palliative discussion integrated into routine care. Healthcare professionals' palliative care education and training are also critical, encompassing knowledge, attitudes, communication skills, and systematic approach, given the challenging time conflicts in clinical practice [39]. Ample evidence could then be used for developing a non-malignancy model for palliative care in HF and interventions, which could eventually accomplish the continuity and shared goals of care for patients with HF through ongoing patient-physician communication. Further studies are also needed to make the provisional efforts for legal ground and policy-making for palliative care of patients with HF.

In conclusion, this study initially showed that the scope of palliative care, using the K-AD model as a vehicle, could be extended to the context of HF in Korea. Poor awareness but moderately positive attitudes toward ADs were noted among the patients with mild HF, with a high acceptance of the K-AD model observed in approximately two-thirds of the patients who completed the K-AD model. This finding supports the feasibility of its use. The major reasons for incompletion were knowledge deficit and decisional difficulty for EOL care. Given the LST Act in action in February 2018 [21], these findings suggest that further exploration of the understanding 
of and attitudes toward ADs of patients with HF and/or their families and their relationships with palliative care with a preparation of an $\mathrm{AD}$ in a larger sample is needed.

\section{KEY MESSAGE}

1. Feasibility of the Korean-Advance Directive (KAD) model was initially supported in heart failure (HF).

2. The major reason for incompletion of advance directives (ADs) in patients with HF was knowledge deficit.

3. End-of-life values of Korean patients with HF were 'comfortable death' and 'no family burdens.'

4. Moderately positive $\mathrm{AD}$ attitudes of patients with HF were associated with more K-AD completion.

\section{Conflict of interest}

No potential conflict of interest relevant to this article was reported.

\section{Acknowledgments}

This work was supported by the Gachon University Gil Medical Center (Grant number: 2016-17).

\section{REFERENCES}

1. Savarese G, Lund LH. Global public health burden of heart failure. Card Fail Rev 2017;3:7-11.

2. Vos T, Flaxman AD, Naghavi M, et al. Years lived with disability (YLDs) for 1160 sequelae of 289 diseases and injuries 1990-2010: a systematic analysis for the Global Burden of Disease Study 2010. Lancet 2012;380:2163-2196.

3. Cubbon RM, Gale CP, Kearney LC, et al. Changing characteristics and mode of death associated with chronic heart failure caused by left ventricular systolic dysfunction: a study across therapeutic eras. Circ Heart Fail 2011;4:396403.

4. Benjamin EJ, Blaha MJ, Chiuve SE, et al. Heart disease and stroke statistics-2017 update: a report from the American Heart Association. Circulation 2017;135:e146-e6o3.

5. Lim SL, Lam CS. Breakthrough in heart failure with preserved ejection fraction: are we there yet? Korean J Intern Med 2016;31:1-14.
6. Liao L, Allen LA, Whellan DJ. Economic burden of heart failure in the elderly. Pharmacoeconomics 2008;26:447-462.

7. Mendis S. Global progress in prevention of cardiovascular disease. Cardiovasc Diagn Ther 2017;7(Suppl 1):S32-S38.

8. Diop MS, Rudolph JL, Zimmerman KM, Richter MA, Skarf LM. Palliative care interventions for patients with heart failure: a systematic review and meta-analysis. J Palliat Med 2017;20:84-92.

9. McIlvennan CK, Allen LA. Palliative care in patients with heart failure. BMJ 2016;353:11010.

10. Adler ED, Goldfinger JZ, Kalman J, Park ME, Meier DE. Palliative care in the treatment of advanced heart failure. Circulation 2009;120:2597-2606.

11. Kim J, Kim S, Shin MS, Jin JO, Kim Y, Lee MO. A context-oriented communication algorithm for advance care planning: a model to assist palliative care in heart failure. J Cardiovasc Nurs 2018;33:446-452.

12. Allen LA, Stevenson LW, Grady KL, et al. Decision making in advanced heart failure: a scientific statement from the American Heart Association. Circulation 2012;125:19281952.

13. Wong FK, Ng AY, Lee PH, et al. Effects of a transitional palliative care model on patients with end-stage heart failure: a randomised controlled trial. Heart 2016;102:11001108.

14. Smith S, Brick A, O'Hara S, Normand C. Evidence on the cost and cost-effectiveness of palliative care: a literature review. Palliat Med 2014;28:130-150.

15. Gelfman LP, Kalman J, Goldstein NE. Engaging heart failure clinicians to increase palliative care referrals: overcoming barriers, improving techniques. J Palliat Med 2014;17:753-760.

16. Butler J, Binney Z, Kalogeropoulos A, et al. Advance directives among hospitalized patients with heart failure. JACC Heart Fail 2015;3:112-121.

17. Dunlay SM, Swetz KM, Mueller PS, Roger VL. Advance directives in community patients with heart failure. Circ Cardiovasc Qual Outcomes 2012;5:283-289.

18. Barclay S, Momen N, Case-Upton S, Kuhn I, Smith E. End-of-life care conversations with heart failure patients: a systematic literature review and narrative synthesis. $\mathrm{Br} \mathrm{J}$ Gen Pract 2011;61:e49-e62.

19. Ziehm J, Farin E, Schafer J, Woitha K, Becker G, Koberich S. Palliative care for patients with heart failure: facilitators and barriers: a cross sectional survey of German health care professionals. BMC Health Serv Res 2016;16:361. 
20. Evangelista LS, Motie M, Lombardo D, Ballard-Hernandez J, Malik S, Liao S. Does preparedness planning improve attitudes and completion of advance directives in patients with symptomatic heart failure? J Palliat Med 2012;15:1316-1320.

21. Ministry of Health and Welfare. Act on hospice and palliative care and decisions on life-sustaining treatment for patients at the end of life 2017, No. 512 [Internet]. Sejong (KR): Korea Ministry of Government Legislation, c2018 [cited 2018 Dec 11]. Available from: http://www.law.go.kr/ 1sEfInfoP.do?lsiSeq=196455\#.

22. Kim SM, Hong YS, Hong SW, Kim JS, Kim KS. Development of a Korean version of an advance directive model via cognitive interview. Korean J Hosp Palliat Care 2013;16:20-32.

23. Kim S, Hong SW, Kim J. Feasibility of the Korean-advance directives among community-dwelling elderly persons. Holist Nurs Pract 2017;31:234-242.

24. Nolan MT, Bruder M. Patients' attitudes toward advance directives and end-of-life treatment decisions. Nurs Outlook 1997;45:204-208.

25. Lee HL, Park YH. Attitudes toward advance directives of older adults using senior centers. J Korean Gerontol Nurs 2014;16:160-169.

26. Levy WC, Mozaffarian D, Linker DT, et al. The Seattle Heart Failure Model (version 2.1) [Internet]. Seattle (WA): University of Washington, 2017 [cited 2018 Dec 10]. Available from: https://depts.washington.edu/shfm/update. php.

27. Levy WC, Mozaffarian D, Linker DT, et al. The Seattle Heart Failure Model: prediction of survival in heart failure. Circulation 2006;113:1424-1433.

28. Pocock SJ, Ariti CA, McMurray JJ, et al. Predicting survival in heart failure: a risk score based on 39372 patients from 30 studies. Eur Heart J 2013;34:1404-1413.

29. Teuteberg JJ, Teuteberg WG. Palliative care for patients with heart failure [Internet]. Washington DC: American
College of Cardiology, 2016 [cited 2018 Dec 10]; Available from: http://www.acc.org/latest-in-cardiology/articles/2016/02/11/08/02/palliative-care-for-patients-withheart-failure.

30. Riley JP, Beattie JM. Palliative care in heart failure: facts and numbers. ESC Heart Fail 2017;4:81-87.

31. Lee MH, Park YH. Factors influencing attitude toward advance directives of older cancer patients. Korean J Adult Nurs 2015;27:449-458.

32. Silveira MJ, Kim SY, Langa KM. Advance directives and outcomes of surrogate decision making before death. $\mathrm{N}$ Engl J Med 2010;362:1211-1218.

33. Kim S, Koh S, Park K, Kim J. End-of-life care decisions using a Korean advance directive among cancer patient-caregiver dyads. Palliat Support Care 2017;15:77-87.

34. Matsui M, Braun KL, Karel H. Comparison of end-of-life preferences between Japanese elders in the United States and Japan. J Transcult Nurs 2008;19:167-174.

35. Krishna LK, Alsuwaigh R, Miti PT, Wei SS, Ling KH, Manoharan D. The influence of the family in conceptions of personhood in the palliative care setting in Singapore and its influence upon decision making. Am J Hosp Palliat Care 2014;31:645-654.

36. Chang MK, Lim MX, Tay K, et al. The influence of evolving confucian beliefs in the selection of proxy decision-makers at the end of life in Singapore. Asian Bioeth Rev 2017;9:117-128.

37. Sim DKL, Tan SH, Tan BC, Neo CL, Wong G. Advance care planning for patients with heart failure in a multiethnic south east Asian cohort. BMJ Support Palliat Care 2013;3:257-258.

38. Hopp FP, Zalenski RJ, Waselewsky D, et al. Results of a hospital-based palliative care intervention for patients with an acute exacerbation of chronic heart failure. J Card Fail 2016;22:1033-1036.

39. Sleeman KE. End-of-life communication: let's talk about death. J R Coll Physicians Edinb 2013;43:197-199. 